\title{
NEW DEVELOPMENT IN CONFOCAL SCANNING OPTICAL MICROSCOPY AND ITS APPLICATION TO THE STUDY OF ELECTRICALLY ACTIVE DEFECTS IN SEMICONDUCTORS
}

\author{
P.S. APLIN \\ H.H. Wills Physics Laboratory, Tyndall Avenue, GB-Bristol BS8 1TL, Great-Britain
}

\begin{abstract}
$\underline{\text { ABSTRACT }}$
A description is given of the design and some early results from a new confocal scanning optical microscope. It is designed for the study of Photoluminescence but has a broad range of potential applications in materials science. The optical system is integrated with a helium cryostat to permit control of specimen temperature from ambient to $2 \mathrm{~K}$. Servomechanisms control the position of the specimen with respect to the focal point of the objective, to eliminate the effects of vibration and drift.
\end{abstract}

\section{INTRODUCTION}

The terms Photoluminescence (PL) and Cathodoluminescence (CL) refer to the study of light released as materials, excited by light or electron beams respectively, decay back to their unexcited states. These techniques have been applied to semiconductor specimens for many years and have contributed greatly to our understanding. For studies in which the specimen is at ambient or even liquid nitrogen temperature, excellent results are achievable from commercially available equipment by either technique. In the case of pure monocrystalline materials, however, we are often interested in resolving information corresponding to energy level changes of a few $\mathrm{meV}$, and this is only possible if the specimen is cooled to a few Kelvin.

Attempts have been made to construct helium cryostats suitable for use with conventional optical microscopes $(1,2)$ but these have had limited success and nearly all low temperature PL work has been done using conventional helium cryostats and simple external lens systems whose functions are to focus the exciting light to a spot on the specimen and as much of the luminescence as possible onto the spectrometer entrance pupil (3). This approach can be modified to permit mapping of luminescence intensity over the specimen surface, but spatial resolution is severely limited by the low aperture optics, disturbance by the boiling helium and the limited rigidity of conventional cryostats.

CL was developed as an adjunct to scanning electron microscopes, so mapping has always been an integral feature. Spatial resolution of CL maps is usually determined by the secondary electron cloud generated within the specimen, further degraded in some cases by mobile excitons. To improve resolution, specimens can be thinned, using the techniques developed for transmission electron microscopy and the very small probe size of an immersion electron lens can be obtained by fitting CL equipment to a 
scanning transmission electron microscope. The disadvantage of this approach is a serious reduction in sensitivity due to quenching of luminescence in the surface layers of the specimen and the extreme difficulty of installing an efficient light collection system in the very restricted space between the specimen holder and the objective lens polepiece.

Refinements of PL that are not possible with CL are excitation luminescence spectroscopy, in which the energy of the exciting photons is the independent variable, and the resolution of spin states by the controlled application of a magnetic or microwave field to the specimen (3). A serious problem with low temperature CL, or any scheme in which the specimen is in a vacuum, arises from the fact that specimen cooling can only be achieved by a solid thermal link to the specimen holder. The link, however, must not stress the specimen because this would introduce spurious luminescence effects.

The instrument to be described was designed to combine the advantages of PL with the ability to map the intensity of spectral features with sub-micron resolution. An overall block diagram, together with a brief description, has already been published (4).

\section{DESIGN CONSIDERATIONS}

\section{General}

As noted above, immersion of the specimen in helium is the preferred means of temperature control. The use of boiling liquid helium is precluded because the bubbles would degrade the resolution of the optical system, and the vibration associated with boiling would increase the problems of mechanical stability. A continuous flow cryostat, using helium gas, is capable of controlling specimen temperature from above ambient to approximately $4 \mathrm{~K}$. Lower temperatures are achieved by immersing the specimen in superfluid helium, which does not boil, but loses heat by evaporation. A suitably designed cryostat can operate in either mode.

Luminescence from semiconductors spans the visible and infrared while imaging detectors of high sensitivity are not commercially available for wavelengths greater than 1 micron. It is therefore necessary to use single element detectors and to scan the specimen point by point to map it. Because of the need to map the intensity of a single spectral feature, Fourier transform spectroscopy of the luminescence cannot be used and we are restricted to a grating spectrometer, possibly fitted with sensitive linear array detectors such as the RCA 32 element photon counting device (5). Such devices give electronic control of spectrometer resolution when mapping and parallel acquisition of spectra.

\section{Microscope Objective}

To maximise spatial resolution and sensitivity, it is essential that both the exciting illumination and the luminescence collection optics have the largest possible numerical aperture and, to avoid restricting the instrument to thin specimens, it must operate in reflection. Thus, a single objective with NA nearly unity is required. Because mobile excitons, in some specimens, can carry energy away from the point of excitation and generate luminescence over an area of several square microns, it is essential that luminescence be collected only from the point on the specimen directly excited. From the foregoing, it is clear that the optical system should take the form of a confocal scanning optical microscope operating in reflection. The entrance pinhole of the confocal system also functions as a spatial filter for the exciting light, while the exit 
pinhole serves also as the entrance pupil of the spectrometer. It is important that the optical system be free from chromatic aberration over the entire spectral range of interest. For those elements handling the exciting beam only, it is sufficient to use conventional achromatic lenses, but for the range of luminescence wavelengths, only mirrors are satisfactory and, in particular, a reflecting objective is essential.

The standard reflecting microscope objective design is that of Schwarzschild. The original design uses aspherical surfaces but Burch (6) showed that an aberration-free design was possible with one spherical and one aspherical surface. Commercially available versions of the Schwarzschild objective use all spherical surfaces and exhibit low aberration if the N.A. is limited to 0.6 or less. In this application, the objective must be inside the cryostat and must therefore function at any temperature from ambient to $2 \mathrm{~K}$. The performance of any multi-element reflecting objective is critically dependent on the relative positioning of the surfaces; a condition difficult to maintain over such a wide temperature range. It was eventually decided to avoid this problem by using a single aspherical surface for the objective; a solution that is not available in conventional imaging microscopy because of the enormous aberration for points off the optical axis. In the system under consideration, the objective is used on axis, and scanning performed by mechanical displacement of the specimen relative to the objective. As the temperature in a helium cryostat is changed, its inner chamber moves a few millimetres with respect to its exterior. The design of the optical system must be such that this movement does not affect the performance of the microscope; a condition most readily met if the light enters and leaves the cryostat as a collimated beam. This implies that the objective should be a paraboloid. A fundamental problem with a reflecting objective is that the centre of the beam is obstructed; by the secondary mirror in multi-element designs and by the specimen in the single element case. The loss of light can readily be made negligible, but in critical applications care must be taken to avoid loss of resolution due to the increased fraction of energy transferred to side lobes in the diffraction pattern. The use of off-axis elements is not a satisfactory solution for this reason.

It is often found necessary to use more than one analytical technique to achieve an understanding of a specimen, and it was therefore decided that this instrument should use the same specimen holders as the transmission electron microscopes in the Department. These are 3mm diameter "cups" so, when allowance is made for the holder and provision for scanning, it is found that the central $10 \mathrm{~mm}$ of the objective is obscured. To reduce the diffraction effect mentioned above to a satisfactory level, an objective diameter of $35 \mathrm{~mm}$ has been chosen. Had the instrument been designed to take much larger specimens, it would have been necessary to use a Burch type modified Schwarzschild objective design.

\section{Specimen Position Control}

To permit rapid specimen changing, it is necessary to use a top loading cryostat design, in which the specimen is attached to the end of a long stalk and lowered to its working position. Even with this arrangement, it is advisable to warm the helium space of the cryostat before opening, if it is to be cooled immediately with a new specimen, to avoid the risk of condensation of atmospheric gas as frost on any of the optical surfaces inside. Hence it is important that the thermal mass in the cold helium space of the cryostat be kept to a minimum, to minimise cooling time. For operation of the cryostat at the lowest temperatures, with the specimen immersed in superfluid liquid helium, it is important that the thermal mass and the heat dissipated in the cold space be 
minimised. Because of this, it is clearly undesirable to put the scan drive mechanism in the cold space and, since optical beam scanning is inappropriate, it is necessary to place the scan drive mechanism at the top of the cryostat and to drive the specimen via its stalk.

As outlined above, the design lacks the stable, rigid linkage between specimen and objective that is generally necessary in any microscope to avoid the effects of vibration and drift. Instead, the position of the specimen with respect to the objective is monitored and the information used both to correct position errors and to ensure that, when mapping, data from the detector is stored in the location corresponding to the actual specimen position and, when acquiring a spectrum, that data is only accepted when the specimen is correctly positioned. In the $X-Y$ plane, normal to the optical axis, the position of the specimen holder relative to the objective is measured by a two axis capacitance displacement system. Electrodes, attached to the objective, and lying outside the light path, are electrically driven to generate an alternating electric field whose amplitude is linearly proportional to distance from the optical axis, and whose phase is a linear function of angular position in the plane. The metallic specimen holder is immersed in this field and is electrically isolated. It is connected to an amplifier in the top of the cryostat and thence to a pair of phase-sensitive detectors whose outputs are proportional to specimen position. This system has spatial resolution of approximately 0.1 micron and time resolution matched to the maximum pixel scanning rate.

The depth resolution of the optical system, determined by the requirements that it be confocal and that the objective NA be large, is approximately 1 micron. It is therefore desirable that control of the specimen position along the optical axis be such that it remain in focus despite irregularities or tilt of its surface with respect to the scanning plane. This can be achieved by monitoring the light reflected back through the optical system from the surface of the specimen. When the surface of the specimen is at the objective focal point, regardless of its angular orientation, reflected light is returned to the source through the entrance pinhole. A sample of this light, deflected by a beamsplitter on the objective side of the pinhole, is analysed by a cylindrical lens and quadrant detector to give a focus error signal which feeds the $Z$ axis specimen position drive. Additional refinements to the electronics enable this system to bring the specimen to focus initially and to cope with temporary loss of signal due to a hole in the specimen or chopping of the exciting beam.

\section{Scanning}

Because the specimen must be mounted strain-free and must not move relative to the holder, the scanning process should not subject it to large acceleration. Mapping the brightness of weak luminescence features with high spatial resolution is only possible with a sufficiently long dwell time on each pixel. These considerations favour slow scanning using a sinusoidal waveform to minimise acceleration. Against this, the slow building of an image is extremely frustrating and time consuming, particularly when the user is simply attempting to locate the appropriate part of the specimen. To obtain the best compromise between these conflicting requirements, it was decided to use a Lissajous scanning pattern because this covers the whole image area quickly at low resolution and then progressively fills in the details. To make efficient use of the scanning time, data must be recorded when the motion is in any direction, and this can only be achieved without loss of resolution if the delay times in the scan position and light detector signals to the computer are matched. Sinusoidal scanning results in non-uniform pixel dwell times; an effect that is further complicated as some pixels 
may be missed on one scan. The solution adopted is that the detector generates pulses at a rate proportional to light intensity and for each pixel, the computer stores both the total number of detector pulses and the total number of clock pulses received as the scan passes it. The intensity of the displayed image is proportional to the ratio of these numbers for each pixel.

From the above, it is clear that the specimen may undergo low frequency sinusoidal motion in the $X Y$ plane and rapid, small amplitude, motion in the $Z$ axis as the automatic focussing system corrects for surface irregularities. It is appropriate to arrange that the $Z$ axis be the axis of the stalk. It follows that the objective should be mounted facing upwards in the cryostat, and that a plane mirror with a central hole be mounted above the objective at an angle of 45 degrees to direct the light through windows in the side of the cryostat.

\section{Optics}

The choice of material for the cryostat windows determines the range of excitation and luminescence wavelengths accessible, and it is natural to seek to maximise this range. However, it was considered desirable to avoid the use of monocrystalline materials such as sapphire because of the complications they would introduce to any possible future applications involving polarisation measurements. An additional consideration is the rapid increase in the radiant heat energy reaching the cold space as the infrared transmission range of the windows is extended toward 10 microns. The material chosen, water free fused silica, has a long wavelength cutoff at approximately 3.5 microns. Two practical details of some importance are that the windows are broadband anti-reflection coated and that they are tilted slightly from normal to the light beam so that any residual reflections are rejected at the exit pinhole.

The luminescence directed to the spectrometer and detector is accompanied by reflected exciting light which may be orders of magnitude more intense and which can generate spurious spectral features because of high order response of the grating or multiple reflections inside the spectrometer. In nearly all cases, it is possible to attenuate the reflected light sufficiently, without affecting the luminescence spectrum, by the choice of a single prefilter. To facilitate this, a wheel carrying some 20 glass filters is attached to the spectrometer to intercept the light beam as it enters. A second source of spurious spectral features is long wavelength radiation from the exciting laser. This is best overcome by the use of a prism in the primary laser beam to deflect energy at unwanted wavelengths away from the entrance pinhole. Finally, Raman lines may be generated in the specimen at sufficiently large shifts from the exciting line to be mistaken for luminescence. To make the necessary distinction, spectra taken with two different exciting wavelengths can be compared.

A conventional broadband metallic beamsplitter is normally most suitable for separating the exciting and reflected beams, but either of two alternatives may be preferred in particular cases. One is a dichroic mirror, which has negligible loss and also functions as a prefilter, but which has serious spectral limitations. The other is a fully reflecting mirror surface of the correct size to illuminate the peripheral annulus of the objective with the exciting light and to direct light reflected from the remainder of the objective to the spectrometer. This improves the ratio of luminescent and Raman light to reflected exciting light entering the spectrometer and, when the transmission of excitation in the specimen by mobile excitons is absent, it gives a small improvement in spatial resolution (7). This is the optimum arrangement for Raman spectroscopy or mapping, provided that adequate exciting power is available. 


\section{CONSTRUCTION}

The cryostat and optical system are mounted on an optical table fitted with an air suspension to reduce vibration. The primary excitation source is a 5 watt Argon ion laser which is used directly at low power and can drive a tuneable laser. The cryostat is a modified version of a standard commercial model, fitted with an added top section containing the three axis specimen drive mechanism. This mechanism tilts the stalk about its top to move the specimen in the $X Y$ plane and moves it axially for $Z$ axis motion. The original version uses piezoelectric stacks for fine movement, together with stepper motors for coarse positioning, but it is proposed to replace these with moving coil actuators.

To minimise the cost and complexity of the cryostat, it is designed to operate as a continuous flow device in the higher temperature range, where the specimen is immersed in gaseous helium, but for low temperature operation, the cryostat is first filled with liquid helium, then rapidly pumped to leave a quantity of superfluid helium whose temperature is controlled in the normal way by slow pumping to control its vapour pressure. A flexible counterflow transfer line carries liquid helium from a storage dewar to the cryostat and returns gaseous helium to a vacuum pump. Its flexibility is sufficient to provide adequate vibration isolation. To achieve the rapid pumping necessary to reach the superfluid helium temperature range quickly, an additional vacuum pump is used, connected to the cryostat via a large diameter flexible pumping line. The second pump does not operate when the microscope is in use, so there is no need to isolate the cryostat from its vibration. Measurement and control of specimen temperature is achieved by a purpose-built unit connected to a platinum resistance thermometer and a heater inside the cryostat, and helium return gas pressure and flow gauges as well as flow control valves. The cryostat radiation shield is cooled by the return gas flow, avoiding the additional complication of a liquid nitrogen system.

All but two of the components in the optical system are standard catalogue items. The exceptions are the mirrors in the cryostat, both of which use aluminium alloy substrates to avoid distortion due to temperature gradients. The planar mirror was fabricated and annealed in the Departmental workshop and sent to an optics firm for final polishing and coating. The paraboloidal mirror was single point diamond turned to our specification by a specialist supplier.

An IBM PS2 model 80 computer is used to control the microscope and to collect, process and display the data. An optical disk drive is used for archiving. Real time processing of images is performed by a digital signal processor chip on a purpose-built interface card, which communicates with the main microscope electronics via a parallel data bus. The electronics directly associated with the microscope has been kept to a minimum. Most of it is the servo loops that control specimen position and scanning.

\section{RESULTS}

The instrument has only recently reached a level of performance sufficient for its use as a research tool. A few images are included to indicate its capabilities. Figure 1 is a rather dirty 10 micron pitch copper grid imaged in reflected light. Figures 3 and 4 are recorded from an experimental strained layer quantum well specimen whose structure is given in Figure 2. The short wavelength region of the luminescence spectrum from this specimen (Figure 3) contains only the peaks from the two narrowest quantum wells; the radiation from the other two quantum wells and from the intervening GaAs 
is at longer wavelengths. The vertical scale is in units of photons per second detected. Figure 4 was obtained by setting the monochrometer to the peak of the luminescence signal from the 20 Angstrom quantum well. The dark lines are due to the quenching of luminescence at strain-induced dislocations where non-radiative recombination is dominant. Both figures 3 and 4 were recorded at a specimen temperature of $4 \mathrm{~K}$.

\section{ACKNOWLEDGEMENTS}

The work was supported by a grant from the SERC. It is a pleasure to acknowledge the work of Drs J C C Day and N W Sidwell, the other members of the team, without whom the instrument would never have been built.

\section{REFERENCES}

1. Heinke, W., Inst. Phys. Conf. Ser., 23 (1975) Ch. 6, p 380.

2. Shirakawa, T., et al, Jpn. J. Appl. Phys., 24 (1985) p 1565.

3. Dean, P.J., Prog. Crystal Growth Charact., 5 (1982) p 89.

4. Aplin, P.S. and Day, J.C.C., Mater. Sci. Eng., B5 (1990) p 329.

5. Trakalo, P., et al, Appl. Optics, 26 (1987) p 3594.

6. Burch, C.R., Proc. Phys. Soc., 59 (1947) p 41.

7. Wilson, T. and Sheppard, C.J.R., (1984) Theory and Practice of Scanning Optical Microscopy, Academic Press, London. 



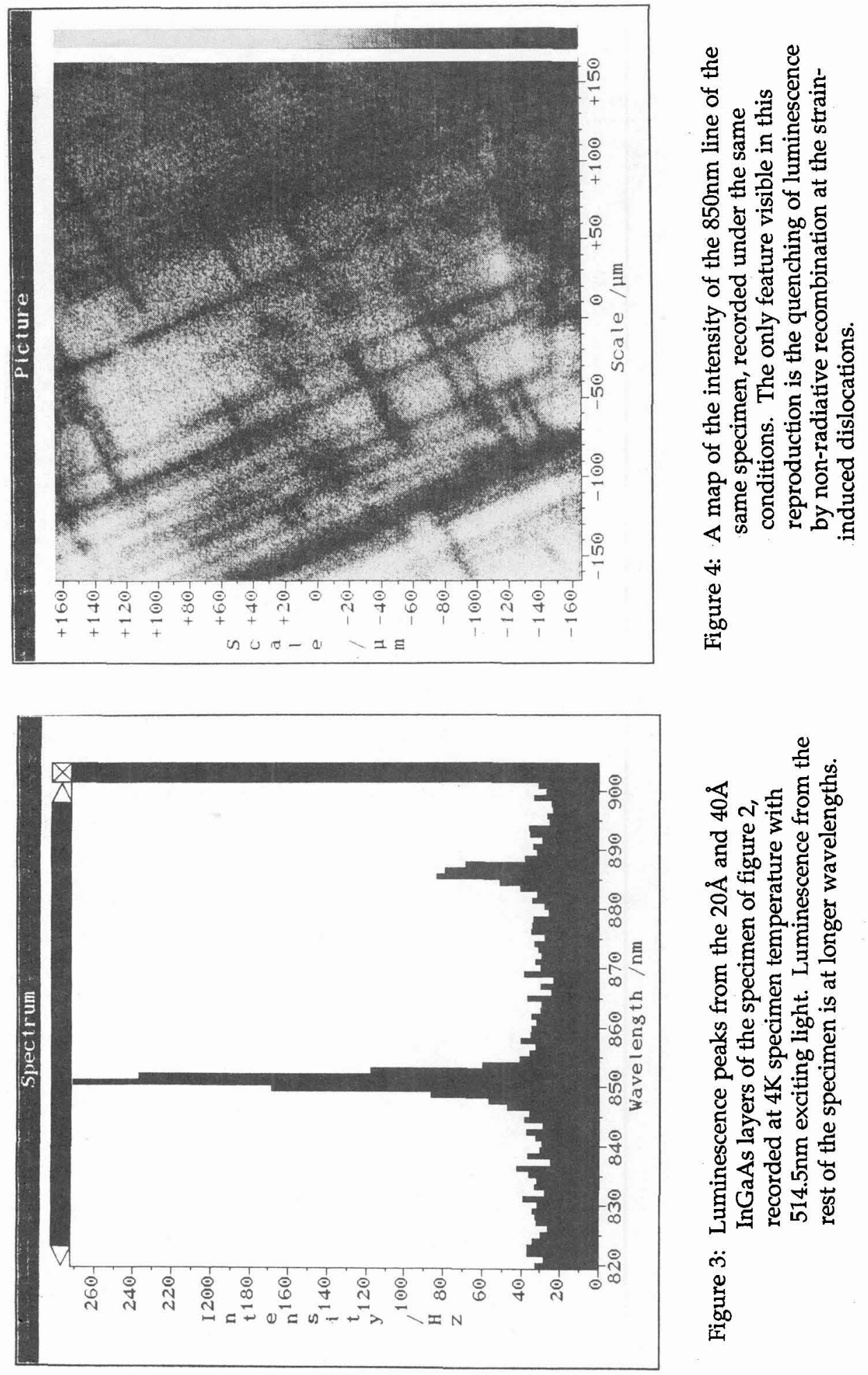